\title{
Gamificación como estrategia de aprendizaje en la formación de estudiantes de Ingeniería*
}

\author{
Gamification as a learning strategy in the training of engineering students
}

\author{
Gustavo Martínez Villalobos ${ }^{a}$, John Fredy Ríos Herrera ${ }^{a}$ \\ ${ }^{a}$ Universidad de Ibagué. \\ gustavo.martinez@unibague.edu.co, John.rios@unibague.edu.co
}

\begin{abstract}
RESUMEN
Las tecnologías de la información y comunicación (TIC) impactan cada día más, consolidando un rol protagónico en procesos educativos y despertando un mayor interés por investigar, buscar y desarrollar medios digitales en la enseñanza para apropiar conocimiento. Así, en este trabajo se presenta el proceso de intervención lúdica y el desarrollo de un videojuego educativo; uno de los productos resultantes de un proyecto de investigación sobre la importancia en el aprendizaje del uso de la gamificación y estrategias pedagógicas asociadas en la formación de estudiantes de ingeniería en un curso de ciencias básicas. El enfoque metodológico utilizado fue cuantitativo de tipo no experimental. El conocimiento adquirido por los participantes se evidenció a través de una evaluación conceptual previa y otra posterior al uso del videojuego en un grupo de 40 alumnos. Los resultados obtenidos indican un mejoramiento actitudinal hacia el curso y un aumento significativo en el desempeño académico de los estudiantes.
\end{abstract}

Palabras claves: Videojuegos, TIC, tecnología educativa, investigación educativa, juegos didácticos.

\begin{abstract}
Information and communication technologies (ICT) impact every day more, consolidating a leading role in educational processes and awakening a greater interest in researching, searching and developing digital media in teaching for appropriate knowledge. Thus, this work presents the process of playful intervention and the development of an educational video game; one of the products resulting from a research project on the importance in learning the use of gamification and pedagogical strategies in the training of engineering students in a basic science course. The methodological approach used was non-experimental quantitative. The knowledge acquired by the participants was evidenced through a conceptual evaluation before and after the use of the video game in a group of 40 students. The results indicate an attitudinal improvement towards the course and a significant increase in the academic performance of the students.
\end{abstract}

Key words: Video games, ICT, educational technology, educational research, didactic games.

\footnotetext{
* Este trabajo forma parte del proyecto investigación "Integración de ludoaprendizaje y REDA para la formación de ingenieros en un curso de ciencias básicas", adscrito al grupo GESE - Universidad de Ibagué.
} 


\section{INTRODUCCIÓN}

En muchas instituciones de educación superior, la formación de ingenieros se ha realizado tradicionalmente a través de clases magistrales y la elaboración de proyectos prácticos de asignatura, que generalmente consisten en simulaciones en pequeña escala. Otras estrategias han sido también utilizadas como videos, estudios de casos y simulaciones por computador con el fin de mejorar y complementar la instrucción. Los profesores han empleado la clase expositiva como herramienta metodológica, para la enseñanza de diversos conceptos, temáticas y teorías con cierta complejidad. En la mayoría de los casos, el docente enmarca su clase en aspectos como la extracción de apartes de un libro, artículo o experiencias, que son transferidos al estudiante. Sin embargo, los problemas por falta de concentración, las dificultades de motivación y aprendizaje, los resultados académicos obtenidos, y el avance de las tecnologías de la información y las comunicaciones (TIC), plantean nuevos retos en investigación y tecnología educativa que requieren también una combinación de nuevas estrategias complementarias a la educación tradicional.

Las nuevas estrategias de aprendizaje en entornos complejos, en los que el conocimiento existente se aplica a nuevos contextos, requiere adoptar actitudes activas y exploratorias (Reig, 2010), como la simulación, el juego, los juegos serios, conocidos en inglés como serious games, el Digital Game-Based Learning o los trabajos sobre ludología (Frasca, 1999). La utilización de plataformas de aprendizaje electrónico (eLearning), blogs, foros virtuales, redes sociales, videochats, videojuegos, y muchas otras herramientas generadas por las TIC han evolucionado hasta el punto de facilitar el uso de juegos para la enseñanza, estableciendo una serie de estrategias que utilizan los juegos como mecanismo de apoyo a las metodologías para la enseñanza de determinados temas de las asignaturas en las diferentes disciplinas. Posiblemente, la gamificación es la herramienta metodológica que más atención ha recibido y se ha considerado más relevante para su implantación en educación (Pérez y Almela, 2018).

La gamificación (también llamada ludificación) es un término que se utiliza para describir aquellas características de un sistema interactivo que tienen como objetivo motivar y comprometer a los usuarios finales a través del uso y la mecánica de estímulos comúnmente incluidos en los juegos. (Zatarain, 2018). Algunos elementos de gamificación utilizados son: puntos, recompensas, progresión, insignias y tablas de posiciones.

El propósito del proyecto, ante la necesidad de explorar otras técnicas educativas que constituyan estrategias claves para responder a los requerimientos de formación en la facultad de ingeniería, de la Universidad de Ibagué, específicamente como aporte académico en un curso de Álgebra Lineal, fue diseñar y combinar juegos didácticos, entre ellos un videojuego, guiados por el profesor, con la intención de aprender a través de la lúdica, i.e., lograr que los estudiantes aprendan jugando, buscando un alto nivel de interacción entre los estudiantes mediante el trabajo en equipo. La presencia de estos recursos tecnológicos y su disponibilidad para un docente del área de ciencias básicas se enmarca dentro de un concepto mayor y más inclusivo que incorpora a la universidad dentro de un concepto de virtualidad como complemento a la presencialidad (Sangrá, 2001).

Asociado también a la era de la información (Castells, 2002), con el conocimiento como elemento central, al carácter multimedia de la información, a los alfabetismos múltiples propios de los nativos digitales (Piscitelli, 2009), surge hoy la necesidad de aprender a comunicar y crear en audio, video, imagen, texto u otro formato. Esta variedad 
de formatos caracteriza a las nuevas culturas participativas (Reig, 2010), inherentes al proceso de convergencia de medios, un aspecto más cultural que tecnológico, propio de la época contemporánea.

\subsection{APRENDIZAJE Y JUEGOS DIDÁCTICOS}

Los juegos didácticos viabilizan el desarrollo de aspectos cognitivos y de actitudes sociales como la iniciativa, la responsabilidad, el respeto, la creatividad, la comunicabilidad, entre otros. (Marín, Ramos, Montes, Hernández y López, 2011) y es posible afirmar que los juegos didácticos son excelentes alternativas a los métodos tradicionales, porque permiten trabajar diferentes habilidades de los estudiantes, conjugando enseñanza y diversión. Estos juegos pueden ser utilizados en la enseñanza de las asignaturas de la ingeniería como elementos motivacionales para el aprendizaje significativo, para conducir al estudiante a formarse a través de nuevas elaboraciones que fomenten su capacidad crítica de la realidad $y$, que de una u otra forma, contribuyan a su autoaprendizaje. El juego puede definirse como un objeto, a partir de sus características esenciales, que genera el sentido y el significado de la acción lúdica (Huizinga, 2003).

Varios autores le otorgan al juego un valor inconsciente respecto al aprendizaje. Aunque se piense que sólo es entretenimiento, una diversión, se juega básicamente porque aprendemos y eso nos gusta (López Raventós, 2016). El juego ha sido catalogado como un medio de desarrollo integral, un vehículo que posibilita aprendizajes de fuerte significación; reduce la sensación de gravedad frente a errores y fracasos; invita a la presencia activa del jugador; desarrolla la creatividad, la competencia intelectual, la fortaleza emocional y la estabilidad personal (Marcano, 2008). Otros investigadores han presentado sus teorías y postulados que relacionan la gran influencia e importancia de las actividades lúdicas en los procesos de enseñanza-aprendizaje. Se reconoce que los juegos generan entretenimiento en sus practicantes, quienes optan por jugarlos movidos por el deseo de divertirse. La clase expositiva no es, en sí misma, divertida, pero la sola invitación a un juego suele tomarse como una invitación a la diversión (Jensen, 2006).

Una de las mejores estrategias pedagógicas para promover el buen desarrollo de las personas, y a su vez propiciar el aprendizaje en diferentes sentidos, es la lúdica. Con esa actitud, los niños y jóvenes pueden mantener, fortalecer, aumentar y desarrollar habilidades que les permiten adaptarse al medio y adquirir conocimientos que les servirán en el desenvolvimiento efectivo en la adversidad (Fernández, 2012). Actualmente, el juego didáctico es valorado como una de las herramientas más efectivas para promover el aprendizaje y transferir el conocimiento por su capacidad de simular la realidad, al ofrecer un escenario para cometer errores y aprender de ellos en la práctica. A este juego con propósito educativo se lo denomina "juego serio". Se trata de juegos que han sido concebidos para el aprendizaje. En este sentido, es necesario distinguir entre juegos serios con propósito educativo, simulaciones y mundos virtuales (Muñoz y Valenzuela, 2014).

\subsection{VIDEOJUEGOS EN EDUCACIÓN SUPERIOR}

Los conceptos anteriores son inherentes a todo tipo de juegos, y se hacen extensivos a los videojuegos, con los cuales se adquieren habilidades adicionales. Desde hace más de tres 
décadas, la tecnología educativa viene evolucionando y actualizándose permanentemente, logrando impactar la comunidad académica con numerosos cambios que hacen más agradable la vida para profesores y estudiantes.

El efecto más claro del poder e impacto formativo de los videojuegos se produce en la adquisición de competencias digitales, en el contexto tecnológico y digital de las sociedades actuales (López Raventós, 2016). La mayoría de los niños accede por primera vez al universo digital a partir de los videojuegos. Algunos videojuegos didácticos, que se usan en las escuelas, permiten la adquisición de nociones y procesos fundamentales, justamente a través de una exploración de la realidad virtual que lleva a los niños desde el aprendizaje de conceptos visoespaciales y la resolución de problemas simples, hasta la adquisición de habilidades complejas (Gramigna y González, 2009).

Un valor relevante que incorporan los videojuegos es que aportan experiencias en modelos o simulaciones basados en la vida real (Gros, 2000); proporcionan un entorno rico para la experimentación en primera persona. El jugador interactúa con el contexto creado, toma decisiones y percibe inmediatamente las consecuencias. Esta actitud positiva que los jóvenes de hoy manifiestan hacia los videojuegos es la que a muchos profesores les gustaría encontrar en sus estudiantes: interés, competitividad, cooperación, búsqueda de soluciones (Padilla, 2011).

El uso de estos videojuegos en las prácticas formativas, a modo de un artefacto o recurso, lleva a una interacción directa de los estudiantes con el juego. La evidencia al respecto indica que esta forma de incorporación ofrece oportunidades reales para desarrollar habilidades vinculadas al desarrollo matemático, creativo, físico, del lenguaje y la alfabetización y del desarrollo personal y social (Gros, 2009).

En el contexto de la educación superior, se ha antepuesto la transmisión de cierto contenido curricular a través de los contenidos más que en el aprendizaje producido por la experiencia de juego (García, Cortés y Martínez, 2011). Se ha potenciado su misión transmisora de cierto conocimiento en detrimento de lo lúdico.

En la literatura especializada, se identifican varios ejemplos de videojuegos utilizados para la enseñanza de diferentes áreas de conocimiento. En el contexto del aprendizaje de matemáticas, se destaca el diseño de un mecanismo para generar juegos adaptativos, de tal forma que los componentes del juego, la secuencia de juegos generada, el escenario de juego y su dificultad se adaptan a las características y a las acciones de cada usuario (Carro, 2002). Otros ejemplos, son resultado de proyectos de investigación educativa, diseñados a medida en universidades y muchos son videojuegos comerciales conocidos (Felicia, 2009), con beneficios formativos para educación primaria, secundaria y superior. Sin embargo, la estrategia de videojuegos en clase no ha sido utilizada comúnmente en la enseñanza tradicional de disciplinas ingenieriles, particularmente en cursos de Álgebra Lineal, y tampoco hay evidencia suficiente de evaluaciones a los procesos que demuestren pedagógicamente las ventajas particulares de la utilización de los videojuegos didácticos.

Los videojuegos no sólo deben verse como un medio que resulta útil para presentar o ambientar a los aprendices respecto a temáticas nuevas, o como un ambiente en el que se resuelven problemas mediante actividades o etapas que vienen configuradas para esto, sino como un ambiente sistémico que muestra una manera de configurar un conjunto de características que pedagógicamente son deseables y esperables (Garrido, 2013). El reconocimiento de que nos encontramos en una sociedad cambiante en la que las formas de 
enseñar deben revisarse y adaptarse a las nuevas formas de aprender, parecen mostrar que es necesario hacer este ejercicio de conexión.

\subsection{DISEÑO DE VIDEOJUEGOS}

Todas las propuestas de diseño de videojuegos educativos deben involucrar en el proceso tanto a profesores como a diseñadores de juegos. Es necesario que estos dos roles participen para poder diseñar de forma adecuada tanto la parte educativa como la de juego. Las propuestas lúdicas comienzan el proceso de diseño con la especificación del contenido educativo. Es la forma adecuada de diseñar juegos educativos, ya que el objetivo principal es el aprendizaje (Padilla, 2011). Una vez detallados los requisitos de aprendizaje, puede componerse la historia del juego más adecuada a los conocimientos que se quieren practicar. Las siguientes características, entre otras, corresponden a la taxonomía de un juego educativo: desafío, curiosidad, control y fantasía (Lepper y Malone, 1987).

En general, los videojuegos deben contener estos elementos: reglas del juego, resultados, esfuerzo, valor, dependencia de los jugadores y consecuencias negociables. A partir de estos elementos, los autores describen su proyecto de entorno de aprendizaje, adicionando las siguientes características: aplicación de TIC, alto nivel de interactividad, soporte para competición, recompensas (insignias), variedad de objetivos, diferentes métodos o caminos para completar el mismo objetivo, opciones para creación de contenidos por parte del usuario, creación, soporte y estilos de aprendizaje colaborativo (Padilla, 2011). En el marco de diseño del videojuego, se adoptaron las actividades del siguiente modelo:

- Arquitectura de soporte: la implementación debe permitir al usuario identificar a cuáles servicios puede acceder después de terminar el diseño del juego.

- Proceso de diseño del juego educativo: las fases, el orden y todos los elementos del proceso; se debe identificar la temática del videojuego y establecer su propósito, identificar y definir conceptos generales de la temática, seleccionar las técnicas más adecuadas según caracterización, incorporar el conocimiento específico en el videojuego, desarrollar pruebas piloto y consolidar el videojuego.

- Modelado del juego, los jugadores y el proceso de aprendizaje: escenario pedagógico, perfil de jugadores, las escenas e interacciones del juego.

- Monitorización, evaluación y análisis: comprobar que el videojuego logra combinar el aspecto de diversión con el aporte al proceso de aprendizaje. Elaborar encuesta de evaluación del videojuego.

Aunque la interacción entre profesores y desarrolladores de videojuegos es muy importante, es quizás el estudiante mismo quien más puede aportar en la generación de ideas para su diseño y creación, dado que puede sentir en carne propia si es interesante, motivante y útil.

Por esta razón, en el proceso de diseño e implementación de este videojuego para el aprendizaje del álgebra lineal, se conformó un equipo interdisciplinar de trabajo integrado, donde los profesores aportaron los conceptos que se querían enseñar, tres estudiantes que ya habían aprobado el curso participaron en el grupo de desarrollo del videojuego, y los estudiantes a quienes se dirigía el juego, a su vez, interactuaron con los diseñadores e investigadores para mejorarlo. 


\section{MATERIAL Y MÉTODOS}

A partir del modelo de proceso, los estándares de diseño para videojuegos educativos y la herramienta de software Unity, el grupo de trabajo desarrolló el recurso digital para apropiación de conocimiento en la asignatura Álgebra Lineal. El contenido de la asignatura escogido para trabajar con gamificación educativa, por solicitud de la Facultad de Ciencias Naturales y Matemáticas, fue el de operaciones con vectores (suma, producto punto, producto cruz, etc.). El videojuego se diseñó para ejecución stand-alone, para ser utilizado como actividad complementaria individual en clase y también usarse de manera extracurricular o en casa. El estudiante accede inicialmente a un juego que le permite realizar tres ejercicios sobre suma de vectores. El jugador debe indicar el resultado y dependiendo de sus respuestas, obtendrá una insignia o medalla de oro, si todas las respuestas son correctas; de plata, si hay una respuesta incorrecta; de bronce, si hay dos respuestas fallidas; y una máscara siniestra si las tres respuestas son erróneas. En esta primera versión del videojuego, se dispone de cuatro niveles de avance. Los ejercicios de las operaciones vectoriales son generados aleatoriamente.

El videojuego se puede integrar en un sistema de gestión del aprendizaje, como Moodle o Claroline, pero una propuesta de valor agregado en este proyecto es que el estudiante puede descargar el archivo desde un servidor Web, como un recurso educativo digital abierto, sin restricciones de acceso, descomprimirlo y ejecutarlo de forma independiente en su computador personal.

\subsection{METODOLOGÍA}

Se utilizó un enfoque cuantitativo de carácter no experimental. Por la facilidad de acceso a la muestra, representativa de la población y seleccionada de manera directa por los investigadores, el tipo de muestreo aplicado es no probabilístico-incidental (Sarabiego, 2004). La pregunta inicial de investigación planteó la posibilidad de diseñar un videojuego educativo que logre el equilibrio en grado de satisfacción, tanto para profesores como para estudiantes, en lo educativo y lo lúdico, respectivamente. Los objetivos planteados para la evaluación del recurso digital se enfocaron en conocer la percepción de los estudiantes acerca de criterios de diseño (como innovación, tiempo, adaptabilidad) y criterios metodológicos (aprendizaje significativo, ampliación de los saberes, impacto en el aprendizaje y en la evaluación de los estudiantes). Después de diseñar el cuestionario de evaluación del videojuego, se realizó una prueba y evaluación por parte de los estudiantes y el análisis de los datos y resultados obtenidos.

\subsection{MUESTRA}

Para la evaluación de la versión definitiva del videojuego, se organizó un curso piloto con una muestra de 40, de los 100 estudiantes universitarios de diferentes programas académicos de ingeniería que lo utilizaron durante el semestre académico. Las valoraciones acerca del videojuego educativo diseñado se tomaron en modo en línea a través de un cuestionario de escala tipo Likert, con cinco opciones de respuesta: 1=Muy de acuerdo, 2=De acuerdo, 3=Indeciso, 4=En desacuerdo y 5=Muy en desacuerdo (ver Tabla 1). Para determinar la consistencia y fiabilidad del instrumento se aplicó la prueba Alfa de Cronbach al cuestionario, obteniendo un valor total de 0,858 . 
Tabla 1. Preguntas y porcentajes obtenidos al aplicar la encuesta al grupo objetivo

\begin{tabular}{|c|c|c|c|c|c|}
\hline Preguntas sobre la percepción del videojuego & 1 & 2 & 3 & 4 & 5 \\
\hline $\begin{array}{l}\text { ¿La actividad del videojuego hizo la clase distinta } \\
\text { de las demás? }\end{array}$ & $26.1 \%$ & $60.9 \%$ & $4.3 \%$ & $8.7 \%$ & $0 \%$ \\
\hline ¿El videojuego es sencillo y fácil de usar? & $21.7 \%$ & $30.4 \%$ & $34.8 \%$ & $13 \%$ & $0 \%$ \\
\hline ¿Está bien estructurado y ordenado? & $21.7 \%$ & $52.2 \%$ & $17.4 \%$ & $8.7 \%$ & $0 \%$ \\
\hline $\begin{array}{l}\text { ¿Su diseño es motivante y causa un impacto } \\
\text { significativo? }\end{array}$ & $8.7 \%$ & $69.6 \%$ & $17.4 \%$ & $4.3 \%$ & $0 \%$ \\
\hline ¿Genera un alto grado de diversión? & $13 \%$ & $47.8 \%$ & $26.1 \%$ & $8.7 \%$ & $4.3 \%$ \\
\hline ¿Promueve el interés hacia la asignatura? & $17.4 \%$ & $56.5 \%$ & $17.4 \%$ & $8.7 \%$ & $0 \%$ \\
\hline $\begin{array}{l}\text { ¿Permite buscar información para resolver y } \\
\text { superar los retos? }\end{array}$ & $26.1 \%$ & $47.8 \%$ & $17.4 \%$ & $4.3 \%$ & $4.3 \%$ \\
\hline ¿El videojuego contribuye al aprendizaje? & $30.4 \%$ & $56.5 \%$ & $8.7 \%$ & $0 \%$ & $4.3 \%$ \\
\hline $\begin{array}{l}\text { ¿Su material de apoyo es útil para ampliar } \\
\text { conocimiento por medio del juego? }\end{array}$ & $21.7 \%$ & $69.6 \%$ & $4.3 \%$ & $0 \%$ & $4.3 \%$ \\
\hline $\begin{array}{l}\text { ¿Estoy satisfecho con mis resultados obtenidos en } \\
\text { el videojuego? }\end{array}$ & $30.4 \%$ & $43.5 \%$ & $13 \%$ & $8.7 \%$ & $4.3 \%$ \\
\hline ¿Me gusta que en la clase se aprenda jugando? & $52.2 \%$ & $39.1 \%$ & $4.3 \%$ & $4.3 \%$ & $0 \%$ \\
\hline $\begin{array}{l}\text { ¿Se deben adicionar otros temas del curso como } \\
\text { nuevos niveles en el videojuego? }\end{array}$ & $47.8 \%$ & $34.8 \%$ & $13 \%$ & $0 \%$ & $4.3 \%$ \\
\hline $\begin{array}{l}\text { ¿Considero importante incluir en las actividades } \\
\text { de clase este videojuego porque ayuda a aprender? }\end{array}$ & $26.1 \%$ & $56.5 \%$ & $13 \%$ & $4.3 \%$ & $0 \%$ \\
\hline $\begin{array}{l}\text { ¿Estoy dispuesto a invertir tiempo en actividades } \\
\text { lúdicas para el aprendizaje? }\end{array}$ & $43.5 \%$ & $47.8 \%$ & $4.3 \%$ & $4.3 \%$ & $0 \%$ \\
\hline
\end{tabular}

1 = Muy de acuerdo; 2 = De acuerdo; 3 = Indeciso 4 = En desacuerdo 5 = Muy en desacuerdo

\section{RESULTADOS}

Un $65.2 \%$ de los estudiantes encuestados corresponde al género masculino y un $34.8 \%$ al femenino, de los programas de Ingeniería Civil, Sistemas, Electrónica, Mecánica e Industrial, que se encuentran entre segundo y cuarto semestre. Con base en la Tabla 1, se interpretaron las características más relevantes del instrumento aplicado al grupo de estudiantes pertenecientes al curso de Álgebra Lineal y los valores porcentuales de cada una de las características a evaluar en el gráfico 1: 
Gráfico 1. Percepción criterios metodológicos y de diseño

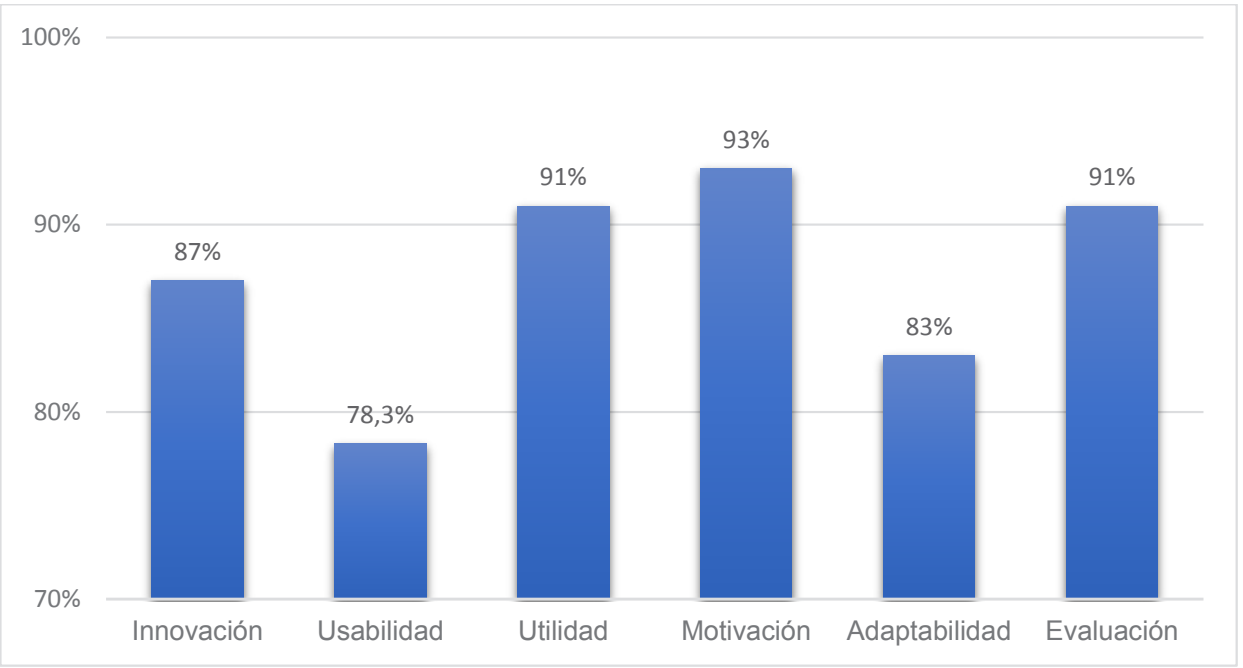

La percepción del $87 \%$ de los estudiantes encuestados denota que la actividad del videojuego en el grupo tuvo un gran impacto al emplearse como una metodología diferente, con el fin de reforzar los temas vistos en clase y su uso como preparación para el examen parcial. El 78.3\% opinó que el diseño del videojuego tuvo una buena aceptación por combinar los temas fundamentales del Álgebra con la historia del videojuego en donde el enfoque es competitivo. Esta percepción indica que el videojuego en general, así como los escenarios, están bien ordenados y estructurados. Más del $90 \%$ de los estudiantes del grupo considera que el videojuego es una herramienta útil para ampliar el conocimiento, reforzar temas de la materia y prepararse para los exámenes, lo cual permite concluir que los componentes diseñados para cada uno de los escenarios fueron adecuados y eficientes, desde la perspectiva de los jugadores. Este resultado evidencia también que, para la mayoría de los estudiantes, tanto la mecánica del juego como sus reglas e instrucciones, fueron explícitas y comprensibles.

A más del $90 \%$ de los estudiantes le parece que jugando es una forma más divertida de aprender, promoviendo la participación y despertando el interés del estudiante en la apropiación de conocimientos en temas fundamentales que contiene la asignatura de Álgebra Lineal y que, en su mayoría, son utilizados en cursos posteriores. Esta percepción positiva de los estudiantes frente al componente de diversión del videojuego resulta satisfactoria para el grupo de investigación, porque permite inferir el potencial de motivación de los juegos en los procesos de enseñanza-aprendizaje. La adición de más temas al curso es de gran interés para más del $80 \%$ de los estudiantes. El juego trataba en su mayoría temas sobre vectores; por lo cual, una propuesta de trabajo futuro será ampliar el contenido del videojuego para aumentar su uso, dada la gran aceptación por parte de los estudiantes. Los jóvenes invierten gran parte de su tiempo en videojuegos. En congruencia con lo anterior, más del $90 \%$ de los estudiantes está de acuerdo en invertir tiempo en actividades lúdicas para el aprendizaje. Se realizó también una confrontación con las notas finales obtenidas 
por estos mismos estudiantes y se obtuvieron los siguientes resultados: El 56.53\% de los estudiantes aprobó la materia con una nota superior o igual a 3.0 y menor a 4.0. El 39.13\% de los estudiantes aprobó la materia con una nota superior o igual a 4.0. El 4,34\%, reprobó la asignatura.

\section{CONCLUSIONES}

Con base en los resultados y la validación de la consecución de los objetivos inicialmente planteados, en este trabajo se pone de manifiesto que los videojuegos educativos van más allá de adquirir conocimientos y desarrollar habilidades. Las respuestas de los estudiantes, que vivenciaron la experiencia de aprendizaje lúdico con el videojuego educativo, confirman los aportes publicados por otros investigadores, citados en este estudio, en el sentido de que los videojuegos requieren la aplicación de los conocimientos adquiridos en la asignatura en la que se trabaja, contribuyen a una mayor motivación, rompen esquemas dentro y fuera del aula y establecen nuevas propuestas didácticas con actividades pedagógicas dinámicas, que hacen más agradable el aprendizaje (Gros, 2009). Aunque se afirma que los escolares ya no encajan bien en el sistema educativo tal como se concibió, por lo que se les puede considerar "nativos digitales" (Prensky, 2001), también es discutible el escaso valor real que otorgan los profesores al uso del juego como medio para el aprendizaje (Gramigna y González, 2009).

Se desarrolló un videojuego educativo para el tema de vectores, de un curso de Álgebra Lineal, que incorpora ciertas características de los juegos. Estas características desencadenan un ciclo que incluye reacciones positivas en la relación estudiante-jugador, tales como el disfrute o interés por la asignatura, comportamientos como una mayor persistencia en la tarea y un sistema de retroalimentación (Turull, 2011). El videojuego fue evaluado por un grupo piloto de estudiantes de ingeniería, que valoró criterios de la fase de diseño, como el contener elementos que hacen la actividad de clase diferente de las demás, el manejo eficiente del tiempo tanto para los estudiantes como para el docente, y también la adaptación del videojuego al currículo, con un trabajo pensado y diseñado para el aprendizaje significativo. También valoraron que el videojuego les permite buscar información para resolver y superar problemas, ampliando así los saberes. Igualmente, fueron valorados los beneficios, efectos y consecuencias en el aprendizaje y evaluación de los estudiantes, a través de un recurso educativo digital abierto que pueden jugar de manera individual en clase o extra clase.

Los videojuegos pueden usarse en el aula para proporcionar diálogo, divertir, motivar, intercambiar opiniones y conocimientos. No es preciso que sean juegos multijugador ya que la interacción se realiza en la propia aula (Gros, 2009). El grupo objetivo también aportó varias sugerencias al videojuego, las cuales fueron asumidas para depurar el diseño. Los cambios o ajustes que surgen durante las pruebas piloto son fundamentales para refinar el videojuego y generar la versión definitiva del recurso digital.

Un medio de impulsar los procesos de innovación en la educación superior, vinculando la tecnología, la pedagogía activa y la institución, es la presentación de propuestas de investigación educativa que proporcionen a la comunidad académica posibilidades de crear su entorno de oportunidad para aprender. Con la realización de este trabajo, se aporta una estrategia de aprendizaje que puede trascender a otras áreas de conocimiento. Finalmente, 
es importante consignar que se aporta también un instrumento útil para el diagnóstico de las percepciones que los estudiantes manifiestan hacia el desarrollo de videojuegos y otras actividades lúdicas para el aprendizaje.

\section{REFERENCIAS BIBLIOGRÁFICAS}

Castells, M. (2002). La Era de la Información. Vol. I: La Sociedad Red. México, Distrito Federal: Siglo XXI Editores.

Carro, R. (2002). Generación de juegos educativos adaptativos. III Congreso Internacional de Interacción Persona - Ordenador, 1664-171.

Felicia, P. (2009). Videojuegos en el aula. Manual para docentes. Bélgica: European school net, (46).

Fernández, T.G. (2012). Ludoaprendizaje como camino de la Resiliencia: plan de desarrollo resiliente (trabajo de grado), Universidad de La Sabana, Bogotá. http://intellectum.unisabana.edu.co/ bitstream/handle/10818/16588/Tany\%20Giselle\%20Fern\%C3\%A1ndez\%20Guayana\%20 $\% 28$ tesis\%29.pdf?sequence $=2 \&$ isAllowed $=\mathrm{y}$.

Frasca, G. (1999). Simulation versus Narrative: Introduction to Ludology. https://www.ludology.org/ articles/VGT_final.pdf

García, F. (2005). Videojuegos: Un análisis desde el punto de vista educativo. En: http://craig.com. ar/biblioteca/Videojuegos.\%20An\%E1lisis\%20desde\%20el\%20punto\%20de\%20vista\%20 educativo\%20-\%20Fern\%E1ndez.pdf

García, M., Cortés, S. \& Martínez, R. (2011). De los videojuegos comerciales al currículum escolar. Las estrategias del profesorado. Revista Icono 14, 9(2), 249-261.

Garrido, J. M. (2013). ¿Por qué los estudiantes juegan con videojuegos de estrategia?: algunos principios para la enseñanza. Revista Electrónica de Investigación Educativa, 15(1), 62-74. Recuperado de http://redie.uabc.mx/vol15no1/contenido-garridojm.html.

Gramigna, A. \& Gonzalez, J. (2009). Videojugando se aprende: renovar la teoría del conocimiento y la educación. [Learning with videogames: Ideas for a Renewal of the Theory of Knowledge and Education]. Comunicar, XVII(33), 157-164. https://doi.org/10.3916/C33-2009-03-007

Gros, B. (2000). La dimensión socioeducativa de los videojuegos. Edutec-e: Revista Electrónica De Tecnología Educativa, 12.

(2009). Certezas e interrogantes acerca del uso de los videojuegos para el aprendizaje. Comunicación, 7(1), 251-264.

Huizinga, J. (2003). Homo ludens. Madrid, España: Alianza Editorial.

Jenkins, H. (2006). Convergence Culture. Where Old and New Media Collide. New York University Press.

Jensen, B. K. (2006). Responding to the enrollment crisis: alternative strategies to increasing student interest in Computer Science. Journal of Computing Sciences in Colleges, 21(4), 8-8.

Leite, J. (2017). Videojuegos Didácticos - Concepto, creación y documentación específica del proceso de diseño y desarrollo en el GDD. Razón y Palabra, 21(97), 94-114.

Lepper, M. R. \& Malone, T.W. (1987). Intrinsic motivation and instructional effectiveness in computerbased education. En: R. E. Snow \& M. J. Farr (Eds.), Aptitude, learning, and instruction: III. Cognitive and affective process analysis (pp. 255-286). Hillsdale, New Jersey: Erlbaum.

López Raventós, C. (2016). El videojuego como herramienta educativa. Posibilidades y problemáticas acerca de los serious games. Apertura, [S.l.], 8(1), 136-151. ISSN 2007-1094. <http://www. udgvirtual.udg.mx/apertura/index.php/apertura/article/view/825/539>.

Marcano, B. (2008). Juegos serios y entrenamiento en la sociedad digital. Revista Electrónica Teoría de la Educación: Educación y Cultura en la Sociedad de la Información, 9(3), 93-107.

Marín, Y., Ramos, A., Montes, J., Hernández, H. y López, J. (2011). Juego didáctico, una herramienta educativa para el autoaprendizaje en la Ingeniería Industrial. [online] Universidad de Córdoba. 
Publicada por la Asociación Colombiana de Facultades de Ingeniería-ACOFI. http://www. acofipapers.org/index.php/acofipapers/2013/paper/viewFile/58/15.

Muñoz, C. \& Valenzuela, J. (2014). Escala de Motivación por el Juego (EMJ): estudio del uso del juego en contextos educativos. Relieve, 20(1), art. 4. doi: 10.7203/relieve.20.1.3878.

Padilla, N. (2011). Metodología para el diseño de videojuegos educativos sobre una arquitectura para el análisis del aprendizaje colaborativo (Tesis doctoral). Universidad de Granada, España. https://dialnet.unirioja.es/servlet/tesis?codigo $=22581$.

Pérez, A. \& Almela, J. (2018). Gamificación transmedia para la divulgación científica y el fomento de vocaciones procientíficas en adolescentes. [Gamification and transmedia for scientific promotion and for encouraging scientific careers in adolescents]. Comunicar, (55), 65-74. https://doi. org/10.3916/C42-2014-06.

Piscitelli, A. (2009). Nativos Digitales. Dieta cognitiva, inteligencia colectiva y arquitecturas de la participación. Buenos Aires: Santillana, Aula XXI.

Reig, D. (2010). El futuro de la educación superior, algunas claves [En línea] REIRE, Revista d'Innovació i Recerca en Educació, 3(2), 98-115. http://www.raco.cat/index.php/REIRE.

Prensky, M. (2001). Digital natives, digital immigrants. En: On the Horizon, 9(5), NCB University Press.

Sangrá, A. (2001). La calidad en las experiencias virtuales de educación superior. http://www.uoc. edu/web/esp/art/uoc/0106024/sangra.html

Sabariego, M. (2004). El proceso de investigación. In R. BISQUERRA, Metodología de la investigación educativa. (pp. 127-163). Madrid: La Muralla.

Turull, S. (2011). Diseño, implementación y evaluación de un juego educativo basado en puzles con Scaffolding orientado a la programación. Ingeniería Técnica en Sistemas Informáticos. Universitat Pompeu Fabra.

Zatarain, R. (2018). Reconocimiento afectivo y gamificación aplicados al aprendizaje de Lógica algorítmica y programación. Revista Electrónica de Investigación Educativa, 20(3), 115-125. https://doi.org/10.24320/redie.2018.20.3.1636. 
